\title{
Pulsed Radiofrequency on Dorsal Root Ganglion Improves Neuropathic Pain-induced Depression in SNI Rats by Regulating Hippocampal BDNF and Neuroinflammation via Spinal IRF8 Inactivation
}

\section{Xueru Xu}

Fujian Provincial Hospital

\section{Zhisen Dai}

Fujian Provincial Hospital

Chun Lin

Fujian Medical University

Fan Lin

Fujian Provincial Hospital

Rongguo Liu ( $\square$ Irgfw88@sina.com )

Fujian Provincial Hospital https://orcid.org/0000-0001-5186-3541

\section{Research}

Keywords: Neuropathic pain, Pulsed radiofrequency, Dorsal root ganglion, Depression, Brain-derived neurotrophic factor, Neuroimmune, Pro-inflammatory factors

Posted Date: April 8th, 2021

DOI: https://doi.org/10.21203/rs.3.rs-379360/v1

License: (c) (i) This work is licensed under a Creative Commons Attribution 4.0 International License.

Read Full License 


\section{Abstract}

Background: Increasing evidence suggests that neuroglia, neuroimmune, and neuroinflammatory processes are involved in the development of nerve injury-induced pain and depression. Interferon regulatory factor 8 (IRF8), a crucial factor for microglial activation, is essential for the development of neuropathic pain. The brain-derived neurotrophic factor (BDNF) and inflammatory mediators (IL-1 $\beta$, IL-6, and TNF- $a$ ) in the hippocampus contribute to the pathophysiology of neuropathic pain-depression comorbidity. Our previous study found that depressive-like behaviors induced by spared nerve injury (SNI) could be improved by applying pulsed radiofrequency (PRF) to the dorsal root ganglion (DRG) (PRF-DRG). However, the anti-depressive mechanisms of PRF-DRG therapy remain largely unknown.

Methods: All rats (except for those in the sham group) were subjected to SNI. The nuclease-free water group and the IRF8 siRNA group were intrathecally injected with nuclease-free water and IRF8 siRNA on days 5 and 6 after SNI, respectively. PRF therapy on the L5 DRG was performed in the PRF group on day 7 after SNI, whereas no PRF current was delivered in the Sham-PRF group. The 50\% paw withdrawal threshold, forced swimming test, and sucrose preference test were performed. The expression levels of spinal IRF8 and hippocampal BDNF were tested by molecular biochemistry, while IL-1 $\beta$, IL-6, and TNF-a were tested by ELISA.

Results: The depressive-like behaviors induced by SNI were remarkably developed in rats, which was indicated by a significant reduction in the sucrose preference rate and prolonged immobility time on day 42 after SNI. Mechanical allodynia and depression-like behaviors of rats with SNI were remarkably improved after PRF-DRG or intrathecal IRF8 siRNA. Spinal IRF8 overexpression, hippocampal BDNF downregulation, and increased hippocampal IL-1 $\beta$ and TNF- $\alpha$ levels were reversed by PRF-DRG, similar to the intrathecal injection of IRF8 siRNA.

Conclusions: PRF-DRG therapy could regulate neuroimmune and neuroinflammatory responses to improve pain-induced depressive-like behaviors. The beneficial effect was correlated with the upregulation of BDNF and inhibition of IL-1 $\beta$ and TNF- $a$ in the hippocampus via spinal IRF8 inactivation.

\section{Background}

Neuropathic pain, such as postherpetic neuralgia, diabetic peripheral neuropathy, and complex regional pain syndrome, is commonly considered an intractable pain condition. Chronic neuropathic pain is prone to induce depression [1, 2], which aggravates disability, poor treatment response, and heavy disease burden [3]. Therefore, a better understanding of the mechanisms underlying neuropathic pain-induced depression is essential for choosing a precise therapy to improve the curative effect.

Studies have demonstrated that neuroinflammation is involved in the neuropathic pain-depression dyad originating from peripheral nerve injury (PNI) [4-6]. Inflammatory cytokines, critical to the neuroimmune response in neuropathic pain, play an important role in the development of depression [7]. In particular, spinal microglia activation, a key component of the neuroimmune system in the development and 
maintenance of neuropathic pain and depression, has been studied for its involvement in the expression of pro-inflammatory cytokines, such as interleukin-1 (IL-1 $\beta$ ), interleukin-6 (IL-6), and tumor necrosis factora (TNF-a) in the central and peripheral nervous system following PNI [7-9]. Thus, modulation of microglial activation and neuroinflammation responses have been proposed for easing neuropathic painassociated depression $[4,10]$.

Notably, it has been proved that interferon regulatory factor 8 (IRF8) can trigger a reactive microglial phenotype $[11,12]$. In our recent studies, we found that reversing excessive spinal IRF8 expression could inhibit microglial activation and improve neuropathic pain and pain-induced depression [13, 14]. Moreover, we demonstrated that the application of pulsed radiofrequency (PRF) on the dorsal root ganglion (DRG) (PRF-DRG) in rats with PNI could ameliorate the neuropathic pain-depression dyad remarkably due to downregulation of spinal IRF8 and microglial activation [13-15]. However, the mechanisms of PRF-DRG via spinal IRF8 to intervene in mood-associated brain regions have not been explored systematically to date.

The hippocampus, a central component of the limbic system, is a crucial mood-regulating region of the brain involved in nociception processing $[16,17]$. The hippocampus plays a critical role in the development of chronic pain and depression [16]. In addition, the evidence suggests that signaling via hippocampal brain-derived neurotrophic factor (BDNF) plays an important role in the pathophysiology of depression and the therapeutic mechanisms of antidepressants [18]. Animal experiments on chronic pain demonstrated that the expression of BDNF in the hippocampus was decreased [19, 20], which further indicated that abnormal hippocampal BDNF might explain the comorbid pain and depression. However, whether restoration of BDNF levels in the hippocampus could be involved in the underlying antidepression mechanism of PRF-DRG remains unclear.

In the present study, we attempted to validate whether hippocampal BDNF and pro-inflammatory factor levels mediated by the inhibited spinal IRF8 contribute to PRF-DRG therapy in improving depressive-like behaviors induced by spared nerve injury (SNI). The results would be valuable for clinicians to prefer PRFDRG to treat chronic pain and depression comorbidity.

\section{Material And Methods}

\section{Animals}

Male Wistar rats weighing 180-200 g were provided by the Shanghai SLAC Laboratory Animal Co., Ltd., China. All animals were kept in plastic cages, at a temperature of $22 \pm 1^{\circ} \mathrm{C}$, relative humidity of $50 \pm 5 \%$, and a light/dark cycle of $12 / 12 \mathrm{~h}$, and were given food and tap water ad libitum. In addition, the animals were habituated to the environment for seven days before starting the experiment.

All animal studies (including the rat euthanasia procedure) were performed in compliance with the institutional animal care regulations and guidelines of Shengli Clinical Medical College of Fujian Medical 
University and conducted according to the Association for Assessment and Accreditation of Laboratory Animal Care (AAALAC) and the Institutional Animal Care and Use Committee (IACUC) guidelines.

\section{Study Design}

Two hundred and forty rats were randomly divided into six groups ( $n=40 /$ group): (1) sham, (2) SNI, (3) Sham-PRF (SPRF), (4) PRF, (5) intrathecal injection of nuclease-free water (NFW), and (6) intrathecal injection of IRF8 siRNA (IRF8 siRNA). All rats (except for those in the sham group) were subjected to SNI of the left sciatic nerve. The rats in the NFW and IRF8 siRNA groups were intrathecally treated with NFW and IRF8 siRNA on days 5 and 6 after SNI, respectively. PRF was applied to the ipsilateral L5 DRG in the PRF group on day 7 after SNI. The basal 50\% paw withdrawal threshold (50\% PWT) was measured before SNI and on days $3,7,8,10,14,21,28,35$, and 42 . Sucrose preference test (SPT) and forced swimming test (FST) were performed, and the expression levels of spinal IRF8, hippocampal BDNF, IL-1 $\beta$, IL-6, and TNF-a were detected on days 21 and 42 (Fig. 1). Based on our previous experiments and other articles $[21,22]$, all biological analyses were repeated five times, with five rats in each biological test method, in each group, and in each time to ensure adequate tissue materials for immunohistochemistry, RT-qPCR, Western blot, and ELISA analysis and to obtain statistical significance. Behavioral measurements were performed with ten rats in each group.

\section{Model of neuropathic pain}

The SNI procedure was performed as described previously [23]. Briefly, the left sciatic nerve and its three terminal branches in the upper lateral thigh were exposed. The tibial and common peroneal nerves were then transected, leaving the remaining sural nerve intact. The muscle and skin were then closed in two layers. In the sham group, only the nerves were exposed, and no cutting or ligation was performed. In all cases, utmost care was taken to neither stretch the nerve or its branches nor affect the intact nerves.

\section{Intrathecal catheters and drug administration}

An intrathecal catheter was inserted as previously described [13]. After anesthesia, a PE-10 polyethylene catheter was implanted between the L4 and L5 vertebrae to reach the spinal cord subarachnoid space before SNI. The rats that showed neurological deficits after catheter implantation were euthanized. IRF8 siRNA (140 pmol; AM16708; Abcam, Cambridge, UK) was dissolved in $20 \mu \mathrm{L}$ of NFW. The rats were intrathecally treated with $20 \mu \mathrm{L}$ IRF8 siRNA or $20 \mu \mathrm{L}$ NFW via the catheter, followed by washing with 10 $\mu \mathrm{L}$ NFW on days 5 and 6 after SNI in the IRF8 siRNA group and NFW group, respectively.

\section{PRF-DRG therapy}

PRF-DRG was conducted as described previously $[13,14]$. The rats were anesthetized with sodium pentobarbital ( $50 \mathrm{mg} / \mathrm{kg}$, i.p.). The left L5 DRG was exposed in the SPRF and PRF groups through laminectomy and facetectomy without injuring the dura mater. An RF electrode (type $20 \mathrm{G}, 5 \mathrm{~cm}$ long, 4 $\mathrm{mm}$ active tip) was placed adjacent to the corresponding DRG via direct visualization using a radiofrequency device (Cosman Medical, Inc., Burlington, MA, USA). PRF waves were applied after performing a nerve stimulation test through muscle contraction of the lower extremities. The parameters 
for stimulating the PRF waves were as follows [24]: pulse rate of $2 \mathrm{~Hz}$, voltage of $45 \mathrm{~V}$, maximum temperature of $42^{\circ} \mathrm{C}$, pulse width of $20 \mathrm{~ms}$, and stimulated time of $6 \mathrm{~min}$. No PRF current was delivered to the SPRF group.

\section{Behavioral measurements}

All behavioral measurements were performed between 8 am and 2 pm by an experimenter blinded to the grouping.

\section{0\% Paw withdrawal threshold (50\% PWT) test}

Rats were placed in individual Plexiglas cells $(10 \times 15 \times 20 \mathrm{~cm})$ on a wire mesh floor for 15 min. Von Frey filaments (Stoelting, Wood Dale, IL, USA) were applied to the plantar surface of the left hind paw using the up-and-down method as previously described [25]. The filament was subjected to a bending force for 3-4 s applied to the hind paw plantar. Avoiding further contact with the filament, quickly turning the head away, scratching the stimulated area, or attacking the filament were considered positive responses. The $50 \%$ paw mechanical withdrawal threshold (50\% PWT) was measured according to the methods reported by Chaplan et al [25].

\section{Sucrose preference test (SPT)}

This test was performed on days 21 and 42, as previously described [26], with minor modifications. A 48$\mathrm{h}$ two-bottle free-choice paradigm was conducted on rats, one of which contained tap water, and the other contained 1\% sucrose solution. After adaptation, the animals were food-and water-deprived for $23 \mathrm{~h}$, followed by the SPT, in which the rats were housed in individual cages exposed to two pre-weighed test solution bottles for $1 \mathrm{~h}$. To avoid possible side effects, the positions of the water and sucrose bottles were changed after $30 \mathrm{~min}$. At the end of the test, fluid consumption was recorded by reweighing the bottles. The sucrose preference $(\%)$ was calculated using the following equation: sucrose preference $(\%)=$ sucrose consumption/(sucrose consumption + water consumption) $\times 100 \%$.

\section{Forced swimming test (FST)}

The forced swimming test was performed on days 21 and 42 after SNI, as described in a previous study [27]. After a minimum of $1 \mathrm{~h}$ of habituation in the test room, the rats were placed in a Plexiglas cylinder (40 cm height $\times 20 \mathrm{~cm}$ diameter) containing water (30 cm depth) at a temperature of $25 \pm 1^{\circ} \mathrm{C}$. The rats were not able to touch the bottom with their hind paws. The FST consisted of two parts: pretesting for 15 min and testing for 5 min, which was conducted $24 \mathrm{~h}$ after the first session. After both sessions, the rats were removed from the water and allowed to dry for $15 \mathrm{~min}$ at $32^{\circ} \mathrm{C}$ before being returned to their home cages. This session was videotaped using a digital camera for subsequent analysis.

\section{Immunohistochemistry (IHC)}

On days 21 and 42, the rats were deeply anesthetized and perfused with $200 \mathrm{~mL}$ of saline, followed by $200 \mathrm{~mL}$ of $0.1 \mathrm{M}$ phosphate buffer ( $\mathrm{pH}$ 7.3) containing 4\% paraformaldehyde (PFA). The L5 spinal 
segments and bilateral hippocampus were removed, post-fixed in 4\% PFA for $24 \mathrm{~h}$, and allowed to equilibrate in $30 \%$ sucrose in phosphate-buffered saline (PBS) overnight at $4^{\circ} \mathrm{C}$. Transverse spinal sections (4 Tran) were cut using a cryostat and collected in $0.01 \mathrm{M} \mathrm{PBS} \mathrm{(pH} \mathrm{7.3).} \mathrm{After} \mathrm{washing} \mathrm{with} \mathrm{PBS,}$ the tissue was pretreated with $0.3 \%$ Triton X-100 with primary antibodies for rabbit anti-rat IRF8 $(6 \mu \mathrm{g} / \mathrm{mL}$; Abcam, Cambridge, UK) and BDNF ( $5 \mu \mathrm{g} / \mathrm{mL}$; Abcam, Cambridge, UK). Next, the slides were incubated with secondary antibodies containing $1 \mu \mathrm{M}$ 4'-6-diamidino-2-phenylindole (Sigma-Aldrich, St. Louis, MO, USA). Using an ECHUNG YM200 microscope with MetaMorph Microscopy Automation and Image Analysis software (Version 6.1 r4, Universal Imaging Corp., Downingtown, PA, USA), digital photomicrographs of 5 randomly selected sections were taken from the left L5 spinal dorsal horn segments and bilateral hippocampus. Quantification of IRF8-positive cells and BDNF-positive cells was performed by calculating DAB positive pixels per area and counted using Image $J$ software (National Institutes of Health Inc., Bethesda, MD, USA).

\section{Quantitative reverse transcription-polymerase chain reaction (RT-qPCR)}

On days 21 and 42 after SNI, the L5 spinal segments and bilateral hippocampi were dissected and immediately frozen in liquid nitrogen. Tissue samples $(100 \mathrm{mg})$ were homogenized in TRIzol reagent (Servicebio Co. Ltd., Wuhan, China). The reaction mixture for the first-strand cDNA synthesis contained the following components: $1 \mu \mathrm{L}$ of Oligo dT, $4 \mu \mathrm{L}$ of $5 \times$ Reaction Buffer, $2 \mu \mathrm{L}$ of $10 \mathrm{mM}$ dNTP Mix, $1 \mu \mathrm{L}$ of RiboLock RNAase inhibitor $(20 \mathrm{U} / \mu \mathrm{L}), 1 \mu \mathrm{L}$ of RevertAi M-MuLV reverse transcriptase $(200 \mathrm{U} / \mu \mathrm{L})$, and total RNA $(2 \mu \mathrm{g})$ were added to RNase-free water in a final volume of $12 \mu \mathrm{L}$. The PCR cycles were as follows: $95^{\circ} \mathrm{C}$ for $10 \mathrm{~min}$, followed by 40 cycles of $15 \mathrm{~s}$ at $95^{\circ} \mathrm{C}$ and $60 \mathrm{~s}$ at $60^{\circ} \mathrm{C}$. The primers used were as follows:

BDNF: 5'-GTGTGACAGTATTAGCGAGTGGG-3' (forward), and 5'-ACGATTGGGTAGTTCGGCATT-3' (reverse).

IRF8: 5'-GCAGGCAAGCAAGACTACAACC-3' (forward), and 5'-CGGGGACGATTCGGTAAACTT-3' (reverse).

ß-actin: 5'-TGCTATGTTGCCCTAGACTTCG-3' (forward) and 5'-GTTGGCATAGAGGTCTTTACGG - 3' (reverse).

The housekeeping gene $\beta$-actin was used as an internal control. Relative expression was calculated using the $2-\triangle \triangle C T$ method: $\triangle C T$ (test) $=C T$ (target, test) $-C T$ (ref, test), $\triangle C T$ (calibrator) $=C T$ (target, cal) $-C T$ (ref, cal), $\triangle \triangle C T=\triangle C T$ (test) - $\triangle C T$ (calibrator).

\section{Western blot (WB)}

On days 21 and 42 after SNI, the L5 spinal cord segments and bilateral hippocampus were collected and frozen at $-80^{\circ} \mathrm{C}$. Proteins were resolved by $10 \%$ sodium dodecyl sulfate-polyacrylamide gel electrophoresis and transferred onto nitrocellulose membranes. The membranes were then blocked with $5 \%$ non-fat milk in PBS ( $\mathrm{pH} 7.4$ ) with Tween $0.05 \%$, followed by incubation with primary antibodies, including IRF8 (AB2165981; 1:500; Abcam, Cambridge, UK), BDNF (AB108319; 1:500; Abcam, Cambridge, 
UK), and $\beta$-actin (\#4967; 1:1000; Cell Signaling Technology, Danvers, MA, USA). Horseradish peroxidase (HRP)-conjugated secondary antibody (MD2142; 1:6000; Medical Discovery Leader Co., Ltd., Beijing, China) was used to detect the primary antibody. The immunoblots were detected using an enhanced chemiluminescence detection system according to the manufacturer's instructions (170-8280; Bio-Rad Laboratories Inc., Hercules, CA, USA). Data were analyzed using Image J software (National Institutes of Health Inc., Bethesda, MD, USA).

\section{ELISA analysis}

On days 21 and 42 after SNI, the bilateral hippocampi were dissected and immediately frozen in liquid nitrogen. Samples were added to PBS (pH 7.4), homogenized by hand or grinders, and centrifuged for 20 min at $3000 \mathrm{rpm}$. The supernatant was then removed. The levels of IL-1 $\beta, I L-6$, and TNF-a were determined using rat IL-1 $\beta$, IL-6, and TNF-a ELISA Kit (Shanghai Mlbio Technology, China) with a commercial reagent kit following the manufacturer's instructions.

All of the above profiles (IHC, WB, RT-qPCR, and ELISA) were detected and counted by an observer blinded to the treatment.

\section{Statistical Analysis}

Statistical analysis was performed using SPSS software (version 20.0; IBM, Armonk, NY, USA). All experimental results were expressed as the mean \pm standard deviation (SD). To analyze the $50 \%$ PWT, we used a repeated-measures two-way ANOVA followed by the LSD test. The sucrose preference rate, forced swimming immobility time, and the levels of IRF8, BDNF, and pro-inflammatory cytokines were analyzed using one-way ANOVA, followed by the LSD test. Statistical significance was set at $p<0.05$.

\section{Results}

\section{PRF-DRG improves the neuropathic pain-induced depressive behaviors in rats with SNI}

First, we examined the changes in the mechanical pain threshold in rats with SNI. Compared with that in the sham group, the $50 \%$ PWT in the SNI group was significantly reduced after $\mathrm{SNI}(P<0.05$, Additional file 1: Table 1, Table 2, and Fig. 2), and reached a peak on day 7 after SNI; thereafter, it maintained a lower level until the end of the observation. The occurrence of mechanical allodynia indicated the successful establishment of the neuropathic pain model. Second, we explored whether depressive behaviors could be induced by neuropathic pain in the present study. On day 42 after $\mathrm{SNI}$, the sucrose preference rate was significantly reduced $(P<0.05$, Fig. 3a), whereas the forced swimming immobility time was remarkably prolonged $(P<0.05$, Fig. $3 \mathrm{~b})$ in the $\mathrm{SNI}$ group compared to the sham group. However, there were no significant differences in the sucrose preference rate and forced swimming immobility time among the six groups on day 21 after SNI. These findings indicated that in the present study, SNI induced depressivelike behaviors in rats on day 42 but not on day 21 after SNI. Third, on day 42 after SNI, the $50 \%$ PWT $(P<$ 0.05, Additional file 1: Table 1, Table 2, and Fig. 2) and sucrose preference rate were increased $(P<0.05$, Fig. 3a), while the forced swimming immobility time was reduced $(P<0.05$, Fig. 3b) in the PRF group 
compared with those in the SPRF and SNI groups. Thus, PRF-DRG could ease the depressive behaviors induced by SNI.

Table 1 Statistical table of 50\% PWT at different times for each group (mean \pm SD)

An additional .txt file shows this in more detail [see Additional file 1]

Table 2 50\%MWT repeated measurement ANOVAs results

\begin{tabular}{|llllll|}
\hline Source of Variation & SS & DF & MS & F value & $P$ value \\
\hline Time x Column Factor & 801.5 & 45 & 17.81 & 23.81 & $P<0.0001$ \\
\hline Time & 1622 & 9 & 180.2 & 240.9 & $P<0.0001$ \\
\hline Column Factor & 4818 & 5 & 963.5 & 1534 & $P<0.0001$ \\
\hline Subject & 33.93 & 54 & 0.6282 & 0.8399 & $P=0.7837$ \\
Residual & 363.5 & 486 & 0.748 & & \\
\hline
\end{tabular}

SS: sum of aquares of deviation from mean; DF: degree of freedom; MS: mean aquare

As can be seen from Table 2 overall, the difference of $50 \%$ PWT in each group was statistically significant, and the difference of $50 \%$ PWT at each time point was statistically significant. At the same time, there was a cross effect between the group and time, so it could be considered that the $50 \%$ PWT was not identical at different time points, and the rate of change at different times in different groups was not identical. In contrast, we can see that sham $>P R F=I R F 8$ siRNA $>S N I=S P R F=N F W$, indicating that the 50\% PWT in the SNI group, PRF group, IRF8 siRNA group, SPRF group, and NFW group were significantly reduced after SNI, and reached a peak on day 7 after SNI, and 50\% PWT was increased in the PRF group and IRF8 siRNA compared with those in the SPRF, SNI, and NFW groups. There were no significant differences in the 50\% PWT between the PRF and IRF8 siRNA groups.

\section{PRF-DRG therapy increases the hippocampal BDNF by inactivating the spinal IRF8 to involve in the improvement of depressive-like behaviors in rats with SNI}

On day 42 after SNI, we found that the $50 \%$ PWT $(P<0.05$, Additional file 1: Table 1 , Table 2 , and Fig. 2$)$ and the sucrose preference rate were increased $(P<0.05$, Fig. 3a), while the immobility time in the FST was reduced $(P<0.05$, Fig. 3b) in the IRF8 siRNA group compared with those in the NFW and SNI groups. Thus, intrathecal injection of IRF8 siRNA could protect against depressive-like behaviors induced by SNI in rats. We next evaluated the expression of BDNF in the hippocampus and IRF8 in the spinal cord of rats by IHC (Fig.4), qRT-PCR (Fig.5), and WB (Fig.6). The levels of hippocampal BDNF were remarkably decreased, and spinal IRF8 was significantly increased in the SNI and NFW groups compared with those in the sham group on days $21(P<0.05)$ and 42 after SNI $(P<0.05)$. We further examined whether hippocampal BDNF expression could be influenced by spinal IRF8 activation. Biochemical analysis 
showed that intrathecal injection of IRF8 siRNA reversed the increased spinal IRF8 $(P<0.05)$ and decreased hippocampal BDNF expression on days $21(P<0.05)$ and $42(P<0.05)$ in the IRF8 siRNA group compared with those in the NFW and SNI groups. Therefore, these results indicate that spinal IRF8 negatively regulates hippocampal BDNF expression.

Similar to the IRF8 siRNA group, biochemical analysis (Fig. 4-6) showed that PRF-DRG therapy inhibited the enhanced expression of spinal IRF8 $(P<0.05)$ while reversing the reduction in hippocampal BDNF expression in the PRF group compared to that in the SPRF group on days $21(P<0.05)$ and $42(P<0.05)$ after SNI. There were no significant differences in the depressive-like behaviors, the levels of spinal IRF8, and hippocampal BDNF between the PRF and IRF8 siRNA groups on days 21 and 42 after SNI. Since spinal IRF8 activation could mediate the reduction of BDNF in the hippocampus, we considered that PRFDRG therapy could mimic the IRF8 siRNA to improve depressive-like behaviors. Thus, PRF-DRG probably increased hippocampal BDNF by inactivating spinal IRF8 to improve pain and depression comorbidity.

\section{PRF-DRG therapy also negatively regulates hippocampal IL-1 $\beta$ and TNF- $\alpha$ by inactivating spinal IRF8 to ameliorate depressive-like behaviors in rats with SNI}

We also evaluated the expression levels of pro-inflammatory factors (IL-1 $\beta$, IL-6, and TNF- $a$ ) in the hippocampus of rats by ELISA (Fig. 7). On day 42 after SNI, the concentrations of hippocampal IL-1 $\beta$ and TNF-a were increased $(P<0.05)$ in the SNI, SPRF, and NFW groups compared with those in the sham group $(P<0.05)$. There were no significant differences in IL-1 $\beta$ and TNF-a among the six groups on day 21 and in IL- 6 among the six groups on days 21 and 42 . These data indicate that hippocampal IL- $1 \beta$ and TNF-a are involved in neuropathic pain-induced depression. Compared with those in the SNI, SPRF, and NFW groups, intrathecal injection of IRF8 siRNA or PRF-DRG treatment resulted in a significant reduction in hippocampal IL-1 $\beta$ and TNF- $\alpha$ concentrations on day 42 after SNI $(P<0.05$, Fig. 7). There were no significant differences in IL-1 $\beta$ and TNF- $\alpha$ levels between the PRF and IRF8 siRNA groups on day 42 . Hence, PRF-DRG therapy could mimic intrathecal injection of IRF8 siRNA to reduce the levels of hippocampal IL-1 $\beta$ and TNF-a. In summary, PRF-DRG decreased hippocampal IL-1 $\beta$ and TNF- $a$ by inactivating spinal IRF8 to participate in pain and depression comorbidity improvement.

\section{Discussion}

In the present study, SNI induced long-lasting pain sensitization and resulted in pain-induced depressive behaviors. In addition, the overexpression of IRF8 in the spinal cord, downregulation of BDNF, and increase of IL-1 $\beta$ and TNF- $\alpha$ in the hippocampus of rats with SNI were detected. In contrast, PRF-DRG or intrathecal injection of IRF8 siRNA relieved mechanical allodynia and depression-like behaviors and reversed the spinal IRF8 overexpression, hippocampal BDNF reduction, IL-1 $\beta$, and TNF- $\alpha$ increase after SNI. These results extend the understanding of the effective mechanisms of PRF on the DRG for treating neuropathic pain-induced depression. To the best of our knowledge, this is the first report on the PRF-DRG to modulate hippocampal neuroinflammation to relieve neuropathic pain-associated depressive behaviors. 


\section{Effects of PRF therapy on neuropathic pain-induced depressive behaviors}

PRF is a minimally neurodestructive therapy used for the treatment of chronic neuropathic pain. Previous studies have suggested that the DRG is an active participant in the development and maintenance of neuropathic pain [28-30]. Targeted modulation of the DRG is considered a critical therapeutic option for neuropathic pain management [31]. The application of PRF on the DRG showed a better analgesic effect than the treatment of the injured peripheral nerve $[15,24,32,33]$. However, research on the treatment of neuropathic pain and depression comorbidity using this method is rare. In the present study, we not only observed the analgesic effects but also focused on improving depressive behaviors induced by SNI.

We observed that the $50 \%$ PWT and sucrose preference percentage were significantly decreased, while the immobility time of FST was significantly increased due to SNI. Our results reproduced the previous findings that animals with PNI could display anhedonia and behavioral despair [34, 35]. In the current study, PRF-DRG therapy resulted in a dramatic increase in 50\% PWT in rats from day 8 to day 42 after SNI, which was consistent with the results reported by Liu et al [36]. These data strengthen the conclusion that the application of PRF-DRG could attenuate neuropathic pain induced by SNI. Notably, the present study reconfirmed our previous findings on the restorative effects of PRF-DRG on neuropathic paininduced depression [13]. Taken together, these results support the antidepressant-like effects of PRF-DRG.

\section{Therapeutic mechanisms of PRF-DRG on pain-depression comorbidity at the levels of spinal cord and hippocampus Effects of PRF-DRG on IRF8 in the spinal cord}

Previous studies have provided evidence that neuroimmune responses contribute greatly to the development of nerve injury-induced neuropathic pain and depression [37]. Microglial cells are resident immune cells that monitor the central nervous system (CNS) and play an essential role in the pathogenesis of many nervous system diseases and conditions, including neuropathic pain and depression. After PNI, microglia cells transform into a reactive phenotype, initiating a progressive series of cellular and molecular changes in the spinal cord. Notably, IRF8 is a critical transcription factor that transforms microglia into a reactive phenotype to release multiple inflammatory cytokines. IRF8 deficiency or PRF-DRG treatment in PNI models can prevent microglial activation and improve pain sensitivity and depressive-like behaviors $[11,13,14]$. The current results reproduced our previous findings that PRF-DRG or intrathecal IRF8 siRNA reversed the overexpression of spinal IRF8 in rats with SNI, thereby contributing to the improvement of depression-like behaviors. Since PRF-DRG can alter the spinal immune environment and exert immunomodulatory and biological effects on cell morphology, synaptic transmission, and pain signals [38], we assumed that the anti-depressive-like effects of PRF-DRG might be achieved by targeting microglial inactivation via the IRF8 signaling pathway at the spinal cord level to prevent neurotransmission between the spinal cord and depression-related brain regions. 


\section{Effects of PRF-DRG on BDNF in the hippocampus}

The hippocampus is a crucial limbic area involved in anxiety, depression, learning, and memory [39]. Recent studies have reported abnormal cytokine expression in the hippocampus of animal models of persistent pain [40]. Additionally, there was a significant association between depressive illness and loss of hippocampal volume in the form of atrophy was reported [41]. BDNF is a major neuronal growth factor in the brain that regulates neurogenesis, neuronal maturation, survival, and synaptic plasticity. Recent studies have demonstrated that BDNF signaling in the hippocampus is significantly correlated with inflammation-related depression [42]. Abnormal BDNF expression changes in the hippocampus are involved in the neuropathology of comorbid neuropathic pain and depression [19,20]. Clinical studies have also shown that BDNF levels are reduced in plasma or brain tissues, especially the hippocampus, in patients with depression. In contrast, an increase in BDNF expression remarkably improved depressive disorders $[43,44]$. Thus, hippocampal BDNF has been suggested as a promising therapeutic target for depression. In our previous study, PRF-DRG exerted antidepressant-like effects in rats with comorbid neuropathic pain and depression. However, the impact of PRF-DRG on BDNF expression in the hippocampus has not been reported.

In the present study, we extended our previous research to focus on the role of BDNF in the hippocampus. Significantly decreased hippocampal BDNF levels in the SNI group were detected on days 21 and 42 . The beneficial effects of PRF-DRG therapy on neuropathic pain-induced depressive behaviors were accompanied by the reversal of the reduced levels of BDNF in the hippocampus. These findings revealed that restoration of hippocampal BDNF expression might exert anti-depressive effects. However, the aforementioned BDNF alterations preceded the changes in sucrose preference rate and immobility time in the FST on day 21. This finding is consistent with clinical observations that report a time lag spanning several weeks before the therapeutic effect. The delayed anti-depressive-like effect may result from multiple complicated factors, and hippocampal BDNF is sensitively triggered and downregulated by PNI. In contrast, in our recent study, the BDNF levels in the nucleus accumbens (NAc) and spinal cord were upregulated in rats with $\mathrm{SNI}[13,15]$. The opposite findings suggested that BDNF was differentially regulated in the hippocampus, spinal cord, and NAc after SNI, and PRF-DRG could negatively regulate BDNF in different regions.

\section{Effects of PRF-DRG on neuroinflammation in the hippocampus}

Neuroinflammation is considered a common mechanism underlying depression comorbidity of chronic pain. A meta-analysis found a significant elevation in TNF-a, IL-6, and IL-1 $\beta$ levels in patients with depression compared to subjects without depression [7]. In addition, inflammatory reactions in discrete brain areas (cingulum, hippocampus, and prefrontal cortex) have been observed in an animal model of neuropathic pain, which affects the descending regulation of pain and the development of pain-induced depression behaviors [45]. In recent years, some studies have revealed that pro-inflammatory factors are markedly increased in the hippocampus of animals with comorbid neuropathic pain and depression [8, 
46]. In addition, affective disturbances in neuropathic pain could be associated with neuroinflammation in the interconnected hippocampal-medial prefrontal circuitry [10].

In this study, we noted a statistically significant increase in IL-1 $\beta$ and TNF-a levels on day 42 after SNI, while IL- 6 was not significantly changed on days 21 and 42 after SNI. These results suggest that IL-1 $\beta$ and TNF- $\alpha$ might be more sensitive than IL- 6 in this model. IL-1 $\beta$ is a pleiotropic cytokine released from glial cells and is involved in a wide variety of disease states associated with immune and inflammatory responses, including neuropathic pain and depression [47]. Another source of IL-1 $\beta$ in the CNS is local amplification via autocrine and/or paracrine mechanisms [48]. The hippocampus is rich in IL-1 $\beta$ binding sites with the highest density [49]. IL-1 $\beta$ signaling applies to the IL-1 receptor type I on the surface of different types of cells and generates signals to the downstream pathways of mitogen-activated protein kinases and nuclear factor kappa-B, leading to global gene regulation [7]. TNF-a is a multifunctional proinflammatory cytokine produced by a variety of cell types in response to inflammatory stimulation, including neurons, glial cells, and immune cells, and plays a regulatory role in the growth, proliferation, differentiation, and apoptosis of nerve cells [50]. Almost all noxious stimulations, including pain, are associated with immune system activation and pro-inflammatory cytokine release. The pro-inflammatory cytokine cascades initially increase TNF- $a$ expression, and TNF- $a$ is the first cytokine to appear in the cascade after stress activation. Animals with elevated TNF-a levels in the hippocampus are prone to chronic pain and depressive behaviors $[19,51]$. There is evidence that sustained nociceptive stimulation leads to increased levels of TNF- $a$ in the hippocampus, which mediates chronic pain and pain-related affective components. Elevated TNF-a levels, in turn, activate the HPA axis, reduce glucocorticoid response, inhibit the production of norepinephrine, deregulate monoamine and glutamate levels, and reduce BDNF in the hippocampus, thus eliciting comorbid pain and depression [17, 51]. These data might explain why hippocampal IL-1 $\beta$ and TNF-a levels were more significant in the current study. Thus, targeting neuroinflammation in the hippocampus is likely to be a new way to treat pain-depression comorbidity. We further observed that the beneficial effects of PRF-DRG therapy were accompanied by a significant reduction in hippocampal IL-1 $\beta$ and TNF-a levels. Therefore, in our study, PRF-DRG regulated neuroimmunity mainly through IL- $1 \beta$ and TNF-a signaling pathways rather than IL-6, thereby alleviating pain-induced depressive-like behaviors. This study revealed a potential anti-inflammatory mechanism, especially the negative regulation of hippocampal IL-1 $\beta$ and TNF- $\alpha$, underlying the therapeutic effects of PRF-DRG therapy for neuropathic pain-depression dyad.

Regulation of hippocampal BDNF and pro-inflammatory cytokines via spinal IRF8 inactivation participates in anti-depressant mechanism of PRF-DRG

Since IRF8 plays a crucial role in activating spinal microglia and anti-depressive-like effects could be achieved by targeting spinal IRF8 signaling, we further investigated the correlation between the expression levels of spinal IRF8 and hippocampal BDNF and pro-inflammatory cytokines. We found that the levels of hippocampal BDNF, IL-1 $\beta$, and TNF- $\alpha$ were reversed by IRF8 siRNA intrathecally in the present study; therefore, we deduced that inactivation of spinal IRF8 expression should mediate hippocampal BDNF, IL-1 $\beta$, and TNF-a levels, which contribute to the improvement of neuropathic pain-associated 
depressive symptoms. In our study, profound antidepressant-like behaviors were conferred by PRF-DRG and IRF8 siRNA. We observed that PRF-DRG therapy had an effect similar to that of an intrathecal injection of IRF8 siRNA. Both treatments reduced the overexpressed spinal IRF8, reversed the reduced hippocampal BDNF, and inhibited the increased IL-1 $\beta$ and TNF-a levels after SNI. No significant differences were detected between the PRF and IRF8 siRNA groups. Therefore, PRF-DRG therapy could mimic IRF8 siRNA to improve depressive-like behaviors.

BDNF protects neurons from damage caused by nerve injury and plays a key role in hippocampal plasticity and memory processes that are disrupted by the dysregulation production of IL-1 $\beta$ [52]. Recent studies have shown that IL-1 $\beta$ inhibits TrkB-mediated BDNF signaling [53] and the levels of transcription factors that regulate BDNF expression [54]. The mechanism by which IL-1 $\beta$ in the hippocampus participates in lipopolysaccharide-induced downregulation of BDNF in mice suggests that IL-1 $\beta$ may be a new target for the treatment of mood disorders [55]. TNF- $a$ has been shown to modulate the exocrine levels of BDNF in human monocytes [56]. TNF-a is most likely to modulate BDNF production in the hippocampus. According to studies on chronic pain and depression, an increase in TNF-a beyond physiological levels decreases BDNF production in the hippocampus [17]. It was found that BDNF levels were decreased in the hippocampus after $\mathrm{SNI}$, and these changes were mediated by microglial cell activation and TNF receptor 1 . It has been suggested that TNF-a regulates BDNF expression and modulates hippocampal synaptic plasticity in the hippocampus through a microglia-dependent mechanism [19]. Moreover, it was suggested that the interaction of BDNF and TNF-a genes might decrease the expression of BDNF in the hippocampus and cause cognitive dysfunction in patients with schizophrenia [57]. However, whether activated spinal IRF8 inhibits BDNF signaling in the hippocampus via weakening hippocampal IL-1 $\beta$ and TNF-a needs to be explored in future research.

In summary, reversing the decreased BDNF and enlarged IL-1 $\beta$ and TNF- $\alpha$ in the hippocampus mediated by the inactivated spinal IRF8 signaling pathway underlies PRF-DRG for improving neuropathic paininduced depression in rats with SNI. These findings also indicate that non-pharmacological treatments could depress spinal microglial activation, thereby mitigating hippocampal neuroinflammation. However, PRF-DRG in the present study only partially alleviated the neuropathic pain-induced depression behaviors, and future investigations are imperative for the other underlying mechanisms to achieve a more satisfactory curative effect.

This study has some limitations. First, we did not use spinal IRF8 activators to further explore whether hippocampal BDNF and pro-inflammatory cytokine levels are closely related to the spinal IRF8 signal pathway. Second, we did not pretreat the SNI rats with BDNF blockers to investigate whether the antidepression effect of the PRF-DRG could be abolished. Third, no significant differences in hippocampal IL6 levels were detected among the groups. These negative results may be related to the different experimental conditions. These limitations need to be addressed in future in-depth studies.

\section{Conclusion}


PRF-DRG therapy plays an immunoregulatory role in weakening neuroinflammation in the spinal cord and hippocampus. Regulation of BDNF, IL-1 $\beta$, and TNF- $\alpha$ in the hippocampus via spinal IRF8 inactivation participates in the therapy of PRF-DRG to ease comorbid pain and depression.

\section{Abbreviations}

IRF8: Interferon regulatory factor 8; BDNF: Brain-derived neurotrophic factor; SNI: Spared nerve injury; PRF: Pulsed radiofrequency; DRG: Dorsal root ganglion; PNI: Peripheral nerve injury; IL-1 $\beta$ : Interleukin-1 $\beta$; IL-6: Interleukin-6; TNF-a: Tumor necrosis factor-邓; AAALAC: Association for Assessment and Accreditation of Laboratory Animal Care; IACUC: Institutional Animal Care and Use Committee; SPRF: Sham-PRF; NFW: Nuclease-free water; 50\% PWT: Basal 50\% paw withdrawal threshold; SPT: Sucrose preference test; FST: Forced swimming test; IHC: Immunohistochemistry; PFA: Paraformaldehyde; WB: Western blot; PBS: Phosphate-buffered saline; qRT-PCR: Quantitative reverse transcription-polymerase chain reaction; CNS: Central nervous system; NAc: Nucleus accumbens

\section{Declarations}

\section{Ethics approval and consent to participate}

All procedures in this study were approved by the Fujian Medical University Experimental Animal Welfare Ethics Committee and were designed to minimize suffering and the number of animals used. All animal studies (including the euthanasia procedure) were performed in compliance with Provincial Clinic College of Fujian Medical University's institutional animal care regulations and guidelines and conducted according to the AAALAC and the IACUC guidelines.

\section{Consent for publication}

All authors agree to the publication of this manuscript.

\section{Availability of data and materials}

The datasets used and/or analyzed in the current study are available from the corresponding author upon reasonable request.

\section{Competing interests}

Each author declares that he or she, or a member of his or her immediate family, has no commercial association that might pose a conflict of interest in connection with the submitted manuscript.

\section{Funding}

This study was supported by the Project of Medical Innovation of Fujian Province, China (2018-CX-6).

\section{Authors' contributions}


XXR designed and performed the experiments, analyzed and interpreted the data, and wrote the manuscript. DZS helped with data collection and interpretation and contributed to critical manuscript revision. LRG conceived the study, obtained funding, and critically revised the manuscript. LC and LF offered lab instruments and participated in data analysis and the revised version. All the authors have read, revised, and approved the final manuscript.

\section{Acknowledgements}

We would like to express our gratitude to the Provincial Clinic College of Fujian Medical University, China, Pain Research Center, Fujian Medical University, China, and Department of Geriatric Medicine, Fujian Provincial Hospital, Shengli Clinical Medical College of Fujian Medical University, China, for their support provided in this study.

\section{Author details}

${ }^{1}$ Department of Pain Management, Fujian Provincial Hospital, Shengli Clinical Medical College of Fujian Medical University, Fuzhou City, Fujian Province, China;

${ }^{2}$ Pain Research Center, Fujian Medical University, Fuzhou City, Fujian Province, China;

${ }^{3}$ Department of Geriatric Medicine, Fujian Provincial Hospital, Shengli Clinical Medical College of Fujian Medical University, Fuzhou City, Fujian Province, China

\section{References}

1. Yalcin I, Bohren Y, Waltisperger E, Sage-Ciocca D, Yin JC, Freund-Mercier MJ, et al. A Time-Dependent History of Mood Disorders in a Murine Model of Neuropathic Pain. Biological Psychiatry. 2011;70:946-53.

2. Gormsen L, Rosenberg R, Bach FW, Jensen TS. Depression, anxiety, health-related quality of life and pain in patients with chronic fibromyalgia and neuropathic pain. Eur J Pain. 2010;14:127.e121-128.

3. Wang XQ, Peng MS, Weng LM, Zheng YL, Zhang ZJ, Chen PJ. Bibliometric Study of the Comorbidity of Pain and Depression Research. Neural Plasticity. 2019; 2019:1-16.

4. Walker AK, Kavelaars A, Heijnen CJ, Dantzer R, Daws LC. Neuroinflammation and Comorbidity of Pain and Depression. Pharmacological Reviews. 2013;66:80-101.

5. Walters ET. Neuroinflammatory contributions to pain after SCl: Roles for central glial mechanisms and nociceptor-mediated host defense. Exp Neurol. 2014;258:48-61.

6. Capuron L, Miller AH. Immune system to brain signaling: Neuropsychopharmacological implications. Pharmacology Therapeutics. 2011;130:226-38.

7. Lees JG, Fivelman B, Duffy SS, Makker PGS, Perera CJ, Moalem-Taylor G. Cytokines in Neuropathic Pain and Associated Depression. Mod Trends Pharmacopsychiatry. 2015;30:51-66. 
8. Yalcin I, Barthas F, Barrot M. Emotional consequences of neuropathic pain: Insight from preclinical studies. Neuroscience Biobehavioral Reviews. 2014;47:154-64.

9. Ho IHT, Chan MTV, Wu WKK, Liu X. Spinal microglia-neuron interactions in chronic pain. J Leukoc Biol. 2020;108:1575-92.

10. Fiore NT, Austin PJ. Are the emergence of affective disturbances in neuropathic pain states contingent on supraspinal neuroinflammation? Brain, Behavior, and Immunity. 2016; 56:397-411.

11. Masuda T, Tsuda M, Yoshinaga R, Tozaki-Saitoh H, Ozato K, Tamura T, et al. IRF8 Is a Critical Transcription Factor for Transforming Microglia into a Reactive Phenotype. Cell Reports. 2012;1:334-40.

12. Masuda T, Iwamoto S, Mikuriya S, Tozaki-Saitoh H, Tamura T, Tsuda M, et al. Transcription factor IRF1 is responsible for IRF8-mediated IL-1 $\beta$ expression in reactive microglia. Journal of Pharmacological Sciences. 2015;128:216-20.

13. Fang $X Y$, Xu XR, Lin XW, Liu RG. Downregulated spinal IRF8 and BDNF in NAC are involved in neuropathic pain-induced depression relief via pulsed radiofrequency on dorsal root ganglion in rat SNI model. Brain Research Bulletin. 2019;146:192-200.

14. Liu RG, Xu XR, Xu YL, Fang XY, Lin XW. Pulsed Radiofrequency on Dorsal Root Ganglion Relieved Neuropathic Pain Associated with Downregulation of the Spinal Interferon Regulatory Factor 8, Microglia, p38MAPK Expression in a CCI Rat Model. Pain Physician. 2018;21:E307-22.

15. Xu XR, Fu SX, Shi XM, Liu RG. Microglial BDNF, PI3K, and p-ERK in the Spinal Cord Are Suppressed by Pulsed Radiofrequency on Dorsal Root Ganglion to Ease SNI-Induced Neuropathic Pain in Rats. Pain Research and Management. 2019; 2019:1-15.

16. Hu XF, Dong YL, Jin XH, Zhang CK, Zhang T, Zhao J, et al. The novel and potent anti-depressive action of triptolide and its influences on hippocampal neuroinflammation in a rat model of depression comorbidity of chronic pain. Brain, Behavior, and Immunity. 2017; 64:180-194.

17. Fasick V, Spengler RN, Samankan S, Nader ND, Ignatowski TA. The hippocampus and TNF: Common links between chronic pain and depression. Neuroscience Biobehavioral Reviews. 2015;53:139-59.

18. Björkholm C, Monteggia LM. BDNF - a key transducer of antidepressant effects. Neuropharmacology. 2016;102:72-9.

19. Liu Y, Zhou LJ, Wang J, Li D, Ren WJ, Peng JY, et al. TNF-a differentially regulates synaptic plasticity in the hippocampus and spinal cord by microglia-dependent mechanisms after peripheral nerve injury. The Journal of Neuroscience. 2017;37:871-81.

20. Liu R, Wu XM, He X, Wang RZ, Yin XY, Zhou F, et al. Contribution of DNA methyltransferases to spared nerve injury induced depression partially through epigenetically repressing Bdnf in hippocampus: Reversal by ketamine. Pharmacology Biochemistry Behavior. 2021;200:173079.

21. Zhou DL, Zhang SQ, Hu L, Gu YF, Cai YM, Wu DQ, et al. Inhibition of apoptosis signal-regulating kinase by paeoniflorin attenuates neuroinflammation and ameliorates neuropathic pain. Journal of Neuroinflammation. 2019; 16. 
22. Xiang HC, Lin LX, Hu XF, Zhu H, Li HP, Zhang RY, et al. AMPK activation attenuates inflammatory pain through inhibiting NF-KB activation and IL-1 $\beta$ expression. Journal of Neuroinflammation. 2019; 16.

23. Decosterd I, Woolf C. Spared nerve injury an animal model of persistent peripheral neuropathic pain. pain. 2000; 87:149-158.

24. Ding YY, Li HX, Hong T, Zhao RJ, Yao P, Zhao GY. Efficacy and Safety of Computed TomographyGuided Pulsed Radiofrequency Modulation of Thoracic Dorsal Root Ganglion on Herpes Zoster Neuralgia. Neuromodulation: Technology at the Neural Interface. 2019; 22:108-114.

25. Chaplan SR, Bach FW, Pogrel JW, Chung JM, Yaksh TL. Quantitative assessment of tactile allodynia in the rat paw. J Neurosci Methods. 1994;53:55-63.

26. Brenes Sáenz JC, Villagra OR, Fornaguera Trías J. Factor analysis of Forced Swimming test, Sucrose Preference test and Open Field test on enriched, social and isolated reared rats. Behav Brain Res. 2006;169:57-65.

27. Porsolt RD, Blavet GA,N, Jalfre M. Behavioural despair in rats a new model sensitive to antidepressant treatments. Eur J Pharmacol. 1978;47:379-91.

28. Mao QX, Yuan JJ, Xiong M, Wu SG, Chen LY, Bekker A, et al. Role of dorsal root ganglion K2P1.1 in peripheral nerve injury-induced neuropathic pain. Molecular Pain. 2017;13:174480691770113.

29. Nascimento Al, Mar FM, Sousa MM. The intriguing nature of dorsal root ganglion neurons: Linking structure with polarity and function. Prog Neurobiol. 2018;168:86-103.

30. Krames ES. The Dorsal Root Ganglion in Chronic Pain and as a Target for Neuromodulation: A Review. Neuromodulation: Technology at the Neural Interface. 2015; 18:24-32.

31. Liem L, van Dongen E, Huygen FJ, Staats P, Kramer J. The Dorsal Root Ganglion as a Therapeutic Target for Chronic Pain. Regional Anesthesia Pain Medicine. 2016;41:511-9.

32. Jiang R, Li P, Yao YX, Li H, Liu RJ, Huang LE, et al. Pulsed radiofrequency to the dorsal root ganglion or the sciatic nerve reduces neuropathic pain behavior, decreases peripheral pro-inflammatory cytokines and spinal $\beta$-catenin in chronic constriction injury rats. Reg Anesth Pain Med. 2019;14:rapm-2018.

33. Das B, Conroy M, Moore D, Lysaght J, McCrory C. Human dorsal root ganglion pulsed radiofrequency treatment modulates cerebrospinal fluid lymphocytes and neuroinflammatory markers in chronic radicular pain. Brain, Behavior, and Immunity. 2018; 70:157-165.

34. Xie ZM, Wang XM, Xu N, Wang J, Pan W, Tang XH, et al. Alterations in the inflammatory cytokines and brain-derived neurotrophic factor contribute to depression-like phenotype after spared nerve injury: improvement by ketamine. Sci Rep. 2017;7:3124.

35. Fu B, Wen SN, Wang B, Wang K, Zhang JY, Weng XC, et al. Gabapentin regulates dopaminergic neuron firing and theta oscillation in the ventral tegmental area to reverse depression-like behavior in chronic neuropathic pain state. Journal of Pain Research. 2018;11:2247-56.

36. Liu Y, Feng Y, Zhang TJ. Pulsed Radiofrequency Treatment Enhances Dorsal Root Ganglion Expression of Hyperpolarization-Activated Cyclic Nucleotide-Gated Channels in a Rat Model of Neuropathic Pain. J Mol Neurosci. 2015;57:97-105. 
37. Torta R, leraci V, Zizzi F. A Review of the Emotional Aspects of Neuropathic Pain: From Comorbidity to Co-Pathogenesis. Pain Therapy. 2017;6:11-7.

38. Koen Van Boxem, N, de Meij A, Kessels M, Van Kleef, Zundert JV. Pulsed Radiofrequency for Chronic Intractable Lumbosacral Radicular Pain: A Six-Month Cohort Study. Pain Med. 2015;16:1155-62.

39. Barkus C, McHugh SB, Sprengel R, Seeburg PH, Rawlins JNP, Bannerman DM. Hippocampal NMDA receptors and anxiety: At the interface between cognition and emotion. European Journal of Pharmacology. 2010;626:49-56.

40. Tyrtyshnaia A, Manzhulo I, Kipryushina Y, Ermolenko E. Neuroinflammation and adult hippocampal neurogenesis in neuropathic pain and alkyl glycerol ethers treatment in aged mice. Int J Mol Med. 2019;43:2153-63.

41. Sheline YI, Liston C, McEwen BS. Parsing the Hippocampus in Depression: Chronic Stress, Hippocampal Volume, and Major Depressive Disorder. Biological Psychiatry. 2019;85:436-8.

42. Zhang JC, Yao W, Hashimoto K. Brain-derived neurotrophic factor (BDNF)-trkB signaling in inflammation-related depression and potential therapeutic targets. Curr Neuropharmacol. 2016;14:721-31.

43. Reinhart V, Bove SE, Volfson D, Lewis DA, Kleiman RJ, Lanz TA. Evaluation of TrkB and BDNF transcripts in prefrontal cortex, hippocampus, and striatum from subjects with schizophrenia, bipolar disorder, and major depressive disorder. Neurobiology of Disease. 2015;77:220-7.

44. Mondal AC, Fatima M. Direct and indirect evidences of BDNF and NGF as key modulators in depression: role of antidepressants treatment. Int J Neurosci. 2018;129:283-96.

45. Burke NN, Finn DP, Roche M. Neuroinflammatory Mechanisms Linking Pain and Depression. Mod Trends Pharmacopsychiatry. 2015;30:36-50.

46. Zong J, Liao XZ, Ren BX, Wang ZP. The antidepressant effects of rosiglitazone on rats with depression induced by neuropathic pain. Life Sciences. 2018;203:315-22.

47. Gui WS, Wei X, Mai CL, Murugan M, Wu LJ, Xin WJ, et al. Interleukin-1 $\beta$ overproduction is a common cause for neuropathic pain, memory deficit, and depression following peripheral nerve injury in rodents. Molecular Pain. 2016;12:174480691664678.

48. Fogal B, Hewett SJ. Interleukin-1 $\beta$ : a bridge between inflammation and excitotoxicity? Journal of Neurochemistry. 2008;106:1-23.

49. Li L, Zou YT, Liu B, Yang RN, Yang JJ, Sun MH, et al. Contribution of the P2X4 receptor in rat hippocampus to the comorbidity of chronic pain and depression. ACS Chemical Neuroscience. 2020;11:4387-97.

50. Pandey GN, Rizavi HS, Zhang H, Ren XG. Abnormal gene and protein expression of inflammatory cytokines in the postmortem brain of schizophrenia patients. Schizophr Res. 2018;192:247-54.

51. Felger JC, Lotrich FE. Inflammatory cytokines in depression: Neurobiological mechanisms and therapeutic implications. Neuroscience. 2013;246:199-229. 
52. Patterson SL. Immune dysregulation and cognitive vulnerability in the aging brain: Interactions of microglia, IL-1 $\beta$, BDNF and synaptic plasticity. Neuropharmacology. 2015;96:11-8.

53. Tong L, Prieto GA, Kramar EA, Smith ED, Cribbs DH, Lynch G, et al. Brain-derived neurotrophic factordependent synaptic plasticity is suppressed by interleukin-1 via p38 mitogen-activated protein kinase. Journal of Neuroscience. 2012;32:17714-24.

54. Guo JJ, Lin PP, Zhao X, Zhang JF, Wei XF, Wang QW, et al. Etazolate abrogates the lipopolysaccharide (LPS)-induced downregulation of the cAMP/pCREB/BDNF signaling, neuroinflammatory response and depressive-like behavior in mice. Neuroscience. 2014;263:1-14.

55. Li MM, Li CL, Yu HJ, Cai XX, Shen XB, Sun X, et al. Lentivirus-mediated interleukin-1 $\beta$ (IL-1 $\beta$ ) knockdown in the hippocampus alleviates lipopolysaccharide (LPS)-induced memory deficits and anxietyand depression-like behaviors in mice. Journal of Neuroinflammation. 2017;14:190.

56. Schulte-Herbrüggen $\mathrm{O}$, Nassenstein $\mathrm{C}$, Lommatzsch $\mathrm{M}$, Quarcoo D, Renz H, Braun A. Tumor necrosis factor-a and interleukin-6 regulate secretion of brain-derived neurotrophic factor in human monocytes. J Neuroimmunol. 2005;160:204-9.

57. Zhang Y, Fang XY, Fan WX, Tang W, Cai J, Song LS, et al. Interaction between BDNF and TNF-a genes in schizophrenia. Psychoneuroendocrinology. 2018;89:1-6.

\section{Figures}

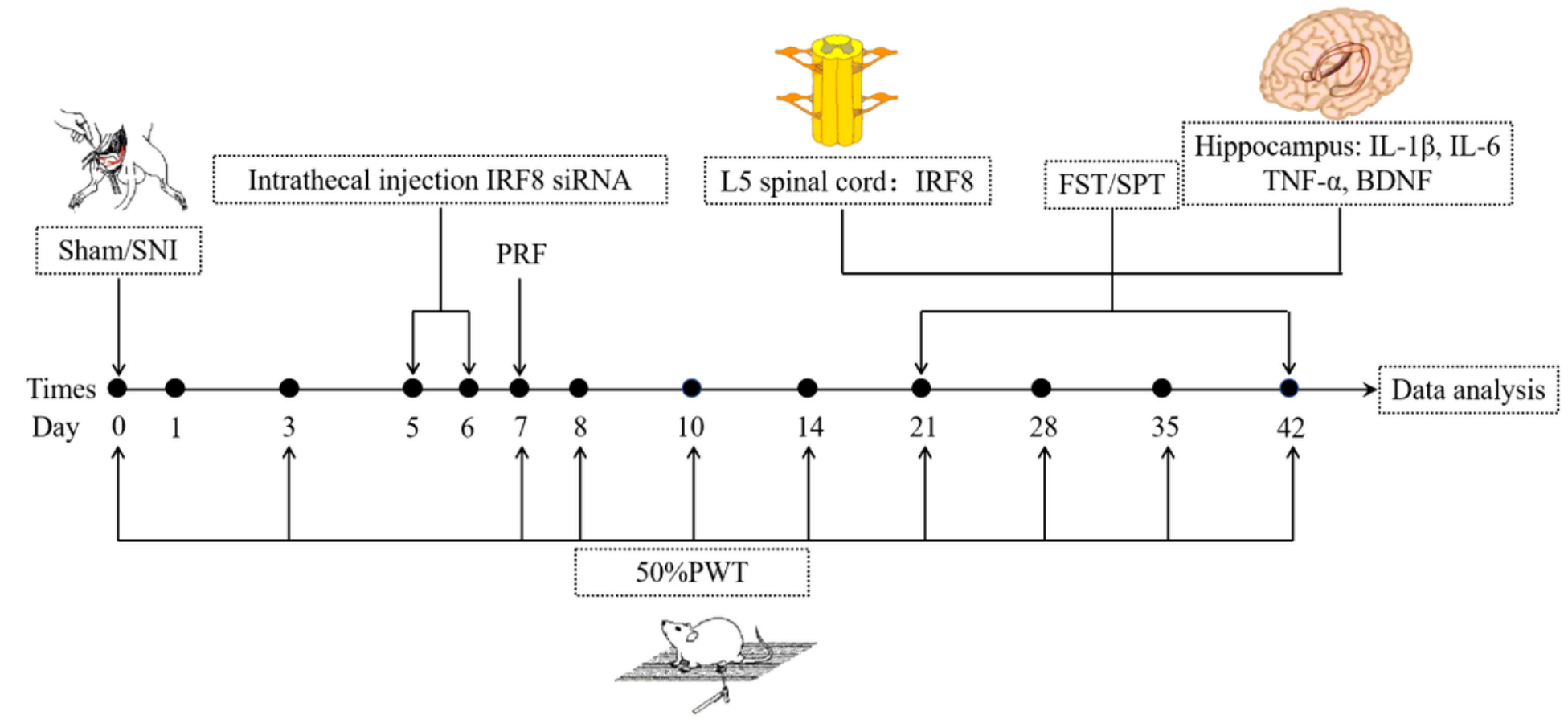

\section{Figure 1}

Treatment groups and design 


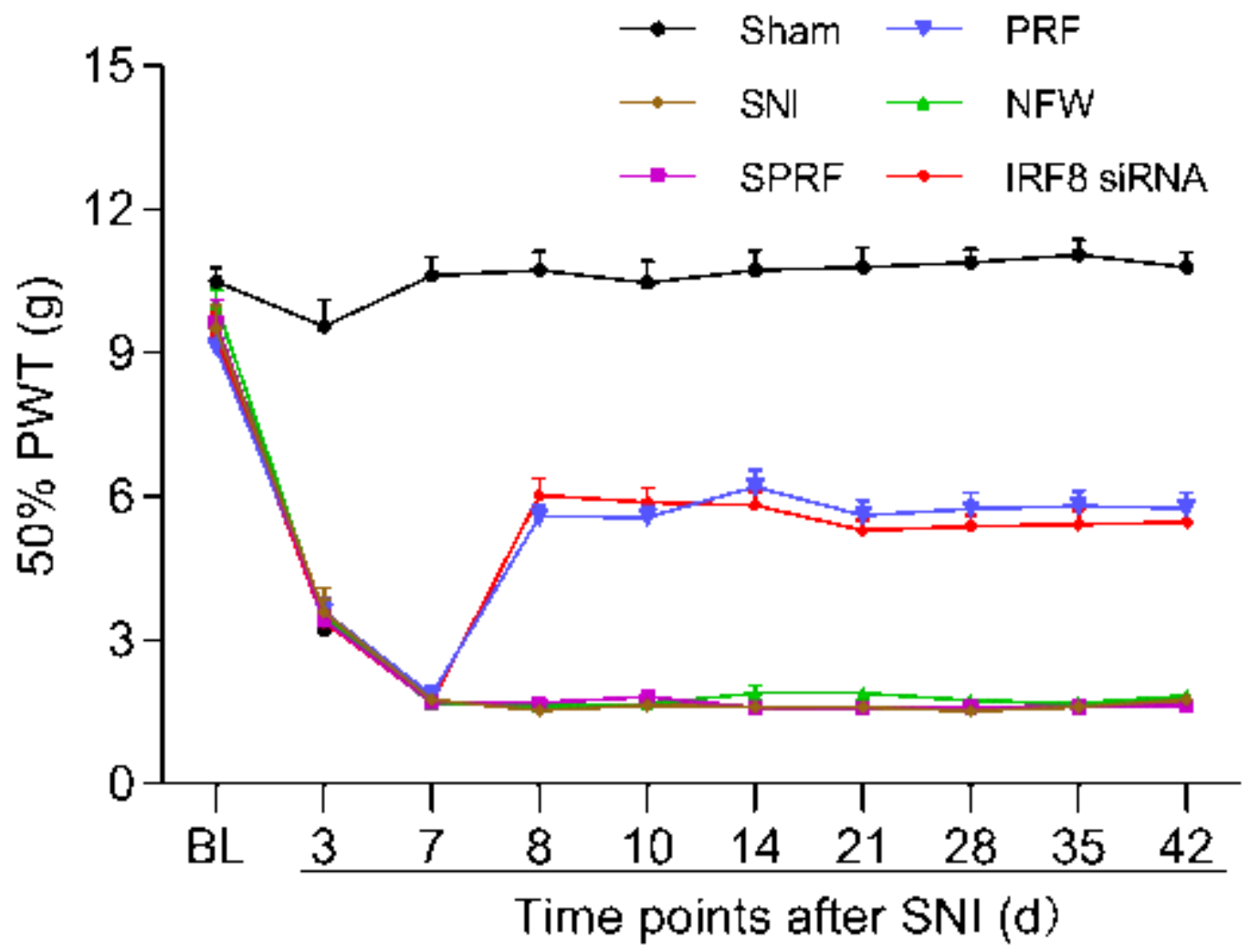

Figure 2

Change chart of $50 \%$ PWT mean \pm SD for each group


Figure 3 
Alterations of sucrose preference rate and forced swimming immobility time on day 42 (a) The reduction of sucrose preference rate induced by SNI on day 42 was partly reversed by intrathecal injection of IRF8 siRNA or PRF-DRG therapy in rats. (b) The increase of immobility time induced by SNI on day 42 was reduced after applying PRF-DRG therapy or intrathecal injection of IRF8 siRNA. Data were expressed as mean $\pm S D$. $* P<0.05$ vs. Sham group; $\# P<0.05$ vs. SPRF group; \&P< 0.05 vs. NFW group. One-way ANOVAs followed by the LSD test, $n=10$ per group.
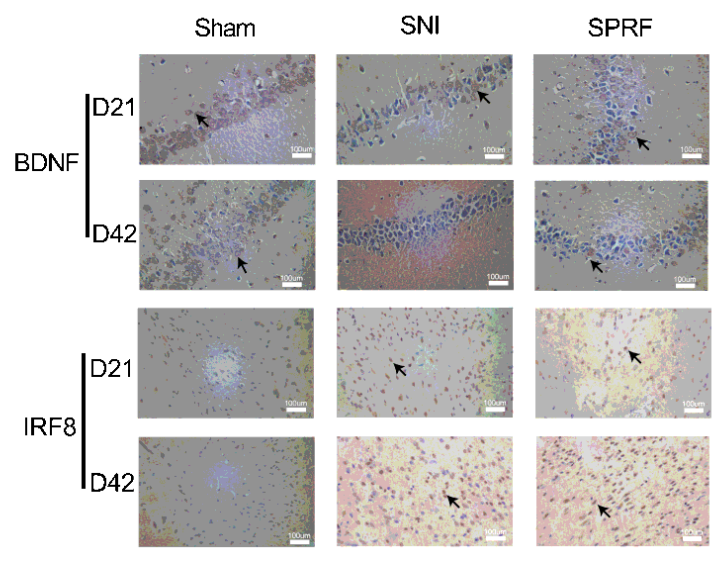

(a)
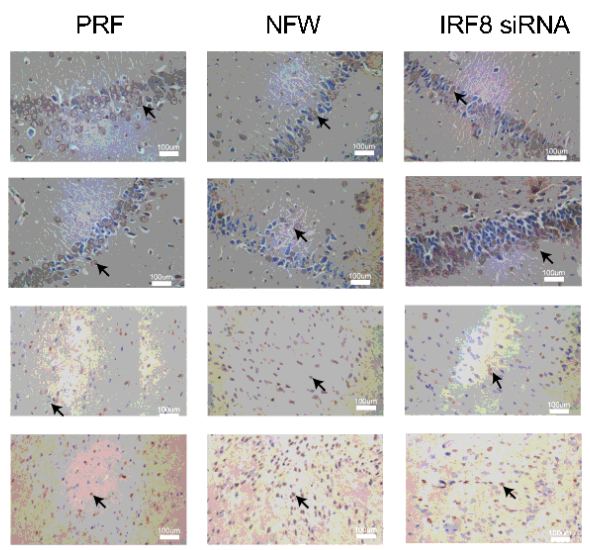

. (b)

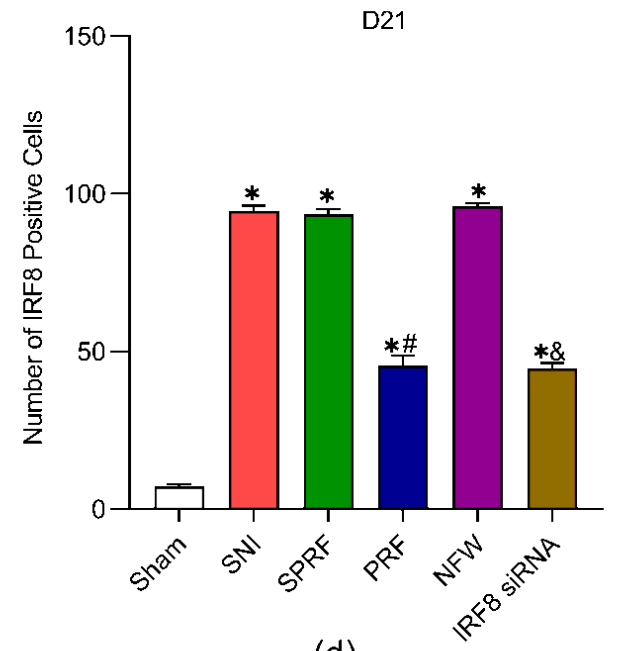

(d)

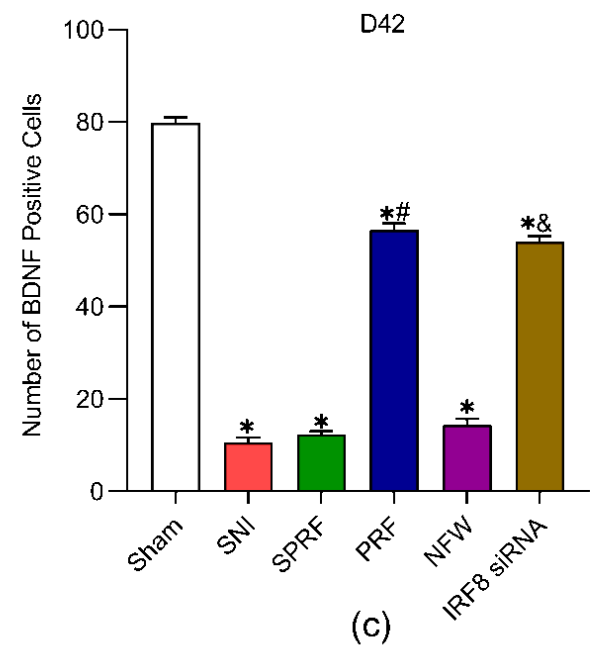

D42

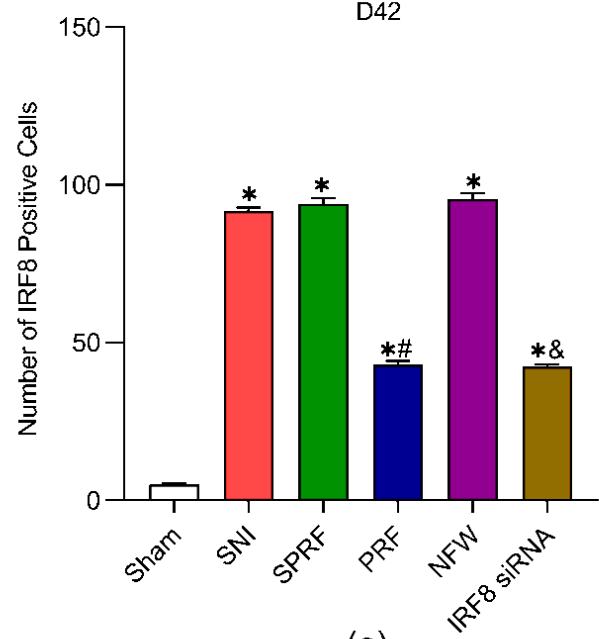

(e)

Figure 4 
Immunohistochemistry analysis of hippocampal BDNF and spinal IRF8 levels on days 21 and 42 (a) Black arrows indicate BDNF-positive and IRF8-positive cells. Scale bar $=100 \mu \mathrm{m}$; magnification $\times 400$. (b,

c) The positive cells of hippocampal BDNF protein induced by SNI on days 21 and 42 were upregulated by intrathecal injection of IRF8 siRNA or PRF-DRG therapy. (d, e) The positive cells of IRF8 protein induced by SNI on days 21 and 42 were reversed by intrathecal injection of IRF8 siRNA or PRF-DRG therapy. Data were expressed as mean $\pm S D$. ${ }^{*} P<0.05$ vs. Sham group; \#P< 0.05 vs. SPRF group; \&P $<0.05$ vs. NFW group. One-way ANOVAs followed by the LSD test, $n=5$ per group.
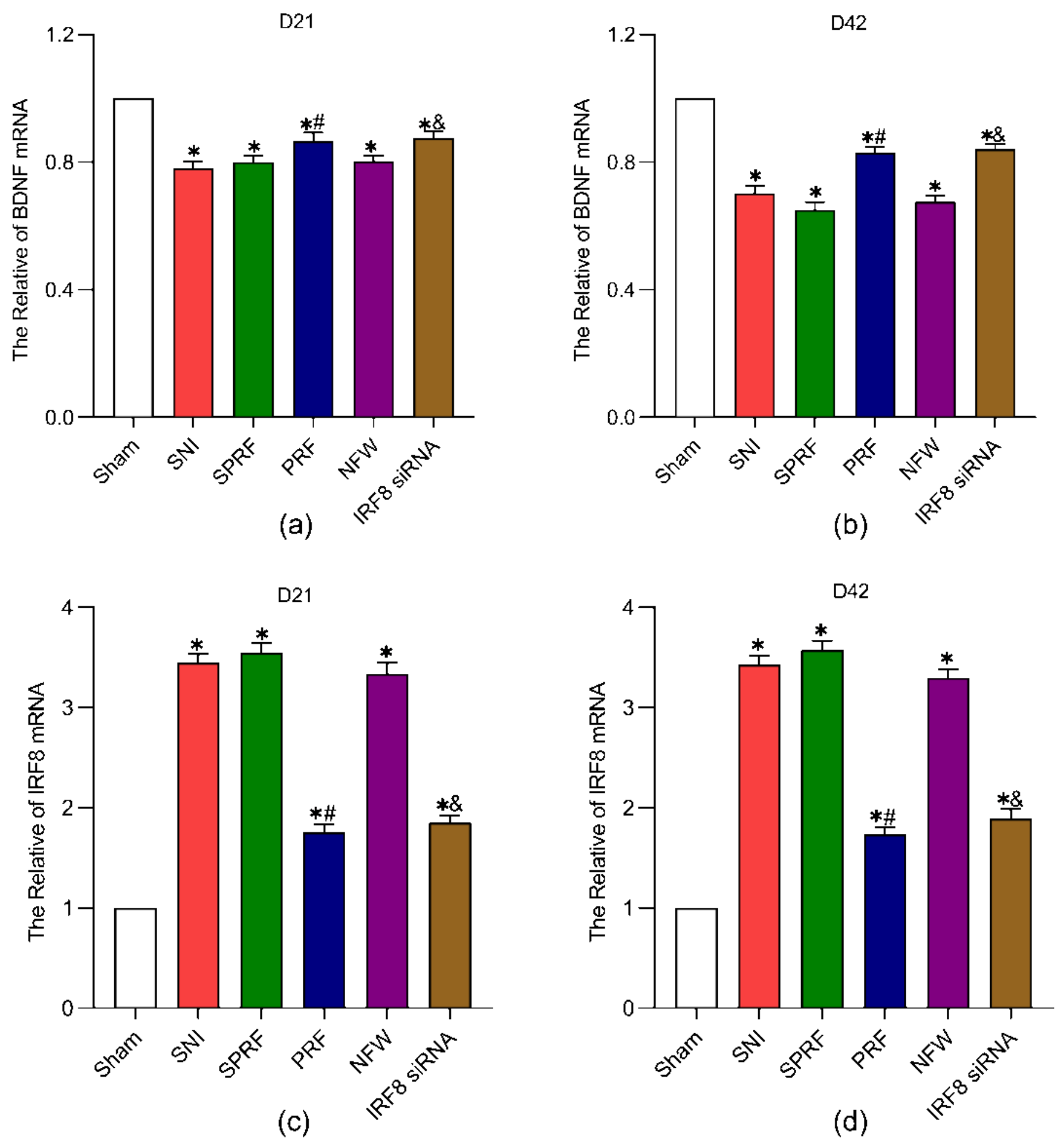


\section{Figure 5}

RT-qPCR analysis of hippocampal BDNF and spinal IRF8 mRNA expression on days 21 and $42(a, b)$ RTqPCR data showed that a reduction of BDNF mRNA levels induced by SNI was reversed by intrathecal injection of IRF8 siRNA or PRF-DRG therapy on days 21 and 42. (c, d) RT-qPCR data revealed that the increase of spinal IRF8 mRNA expression was downregulated by intrathecal injection of IRF8 siRNA or PRF-DRG therapy in SNI rats on days 21 and 42. Data were expressed as mean $\pm S D$. ${ }^{*} P<0.05$ vs. Sham group; $\# P<0.05$ vs. SPRF group; \&P< 0.05 vs. NFW group. One-way ANOVAs followed by the LSD test, $n=5$ per group. 


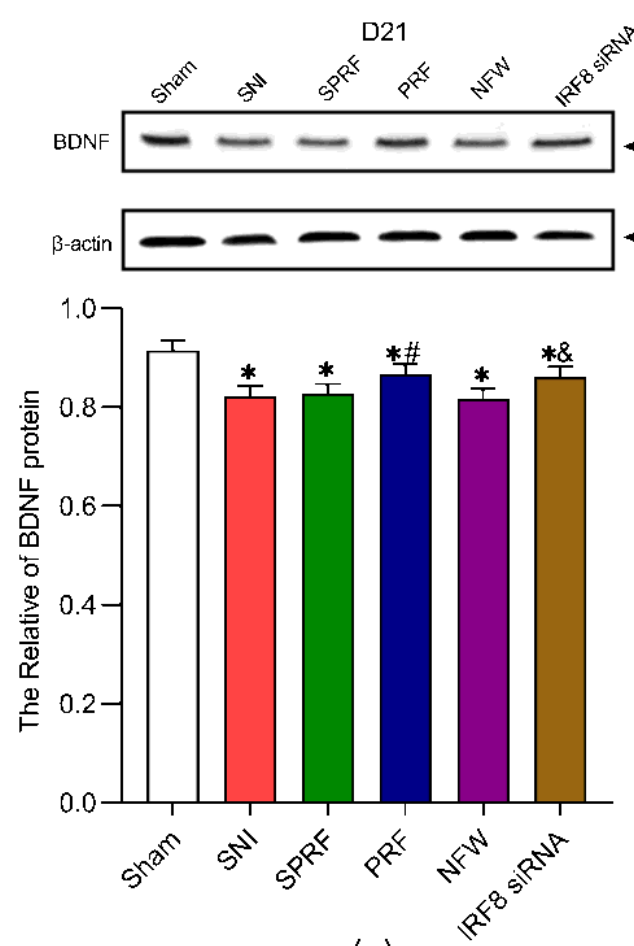

(a)

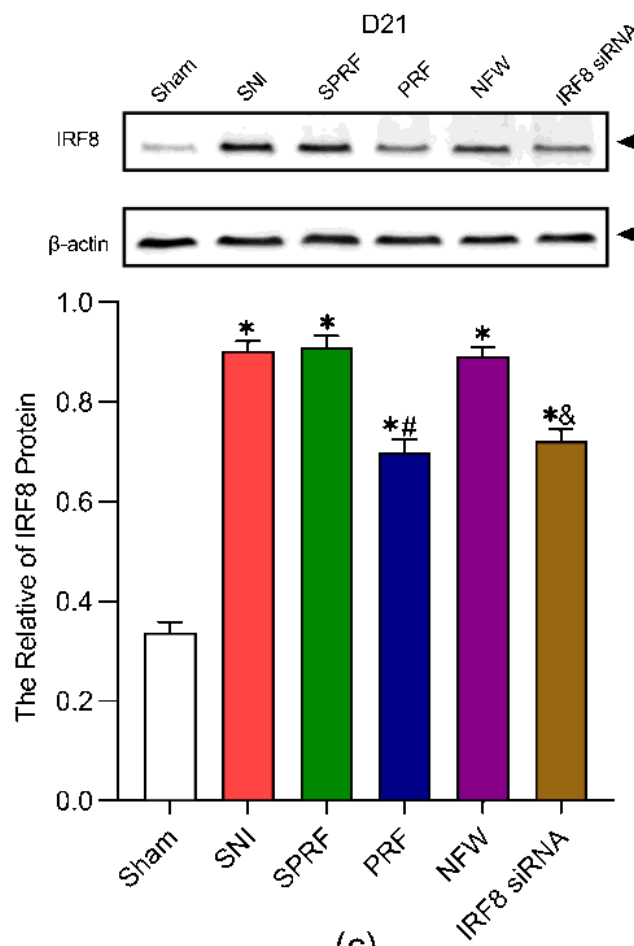

(c)
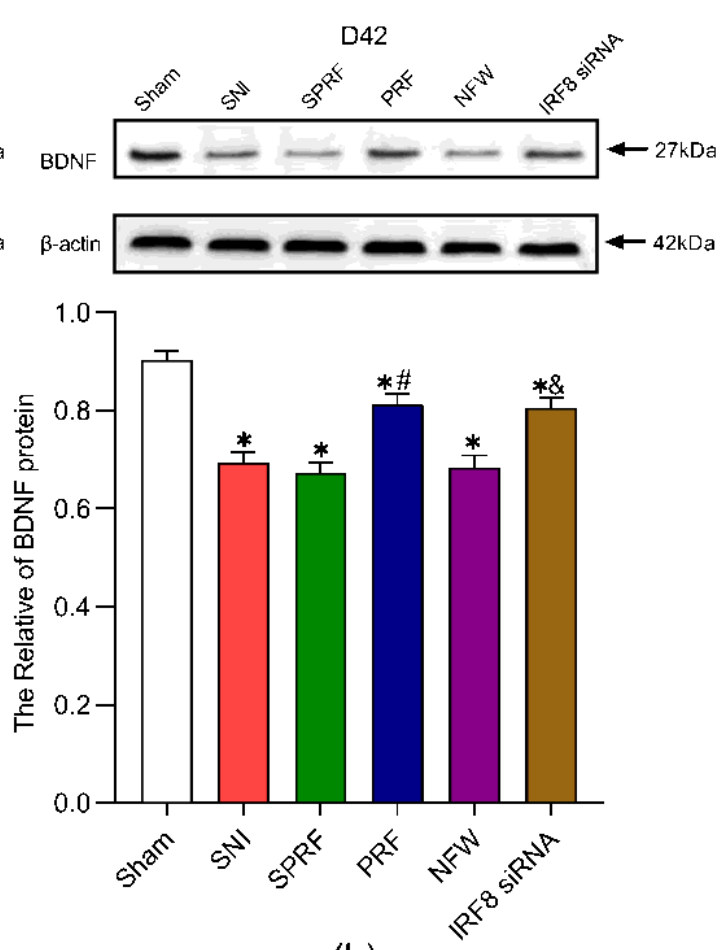

(b)
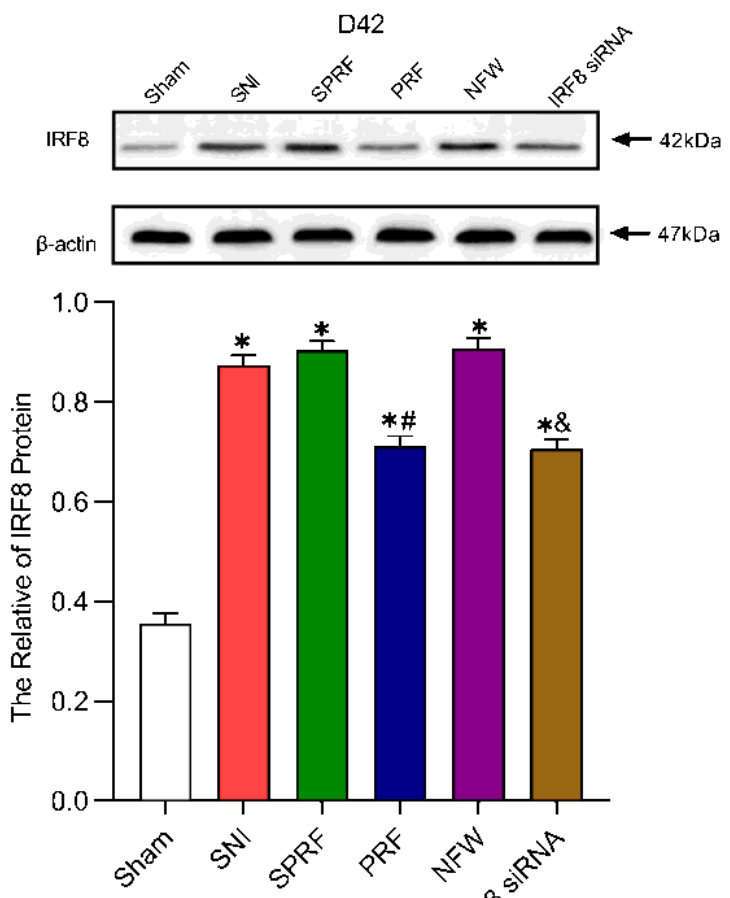

(d)

\section{Figure 6}

WB analysis of hippocampal BDNF and spinal IRF8 protein expression on days 21 and $42(a, b)$ Regarding the levels of hippocampal BDNF protein, its reduction due to SNI on days 21 and 42 were reversed by intrathecal injection of IRF8 siRNA or PRF-DRG therapy. (c, d) The overexpression of spinal IRF8 induced by SNI was remarkably down-regulated by intrathecal injection of IRF8 siRNA or PRF-DRG 
therapy. Data were expressed as mean \pm SD. ${ }^{*} P<0.05$ vs. Sham group; $\# \mathrm{P}<0.05$ vs. SPRF group; \&P< 0.05 vs. NFW group. One-way ANOVAs followed by the LSD test, $n=5$ per group.
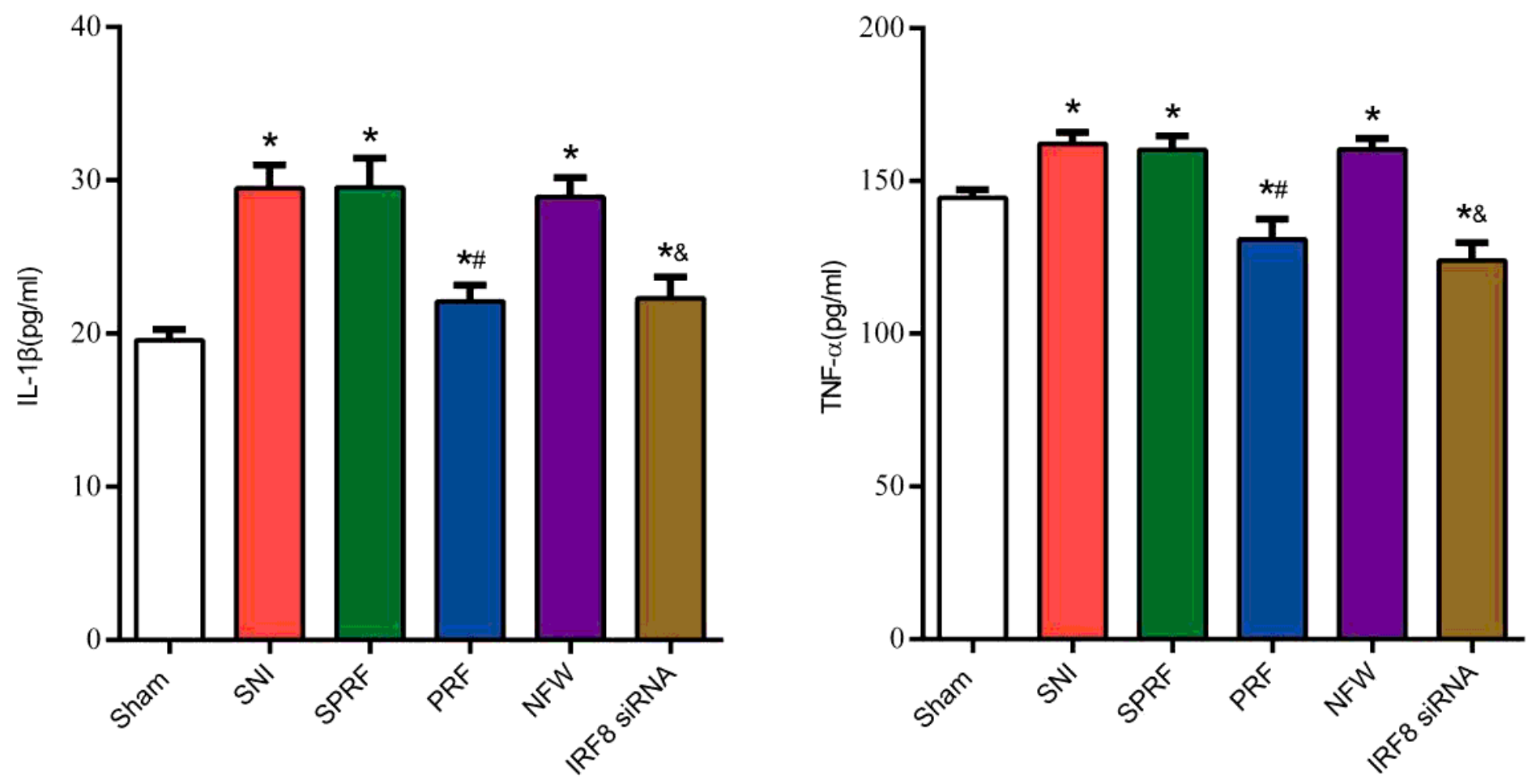

\section{Figure 7}

ELISA analysis of concentration of hippocampal IL-1 $\beta$ and TNF- $\alpha$ on day 42 after SNI The hippocampal IL-1 $\beta$ and TNF- $\alpha$ increase induced by SNI was reversed by intrathecal injection of IRF8 siRNA or PRF-DRG therapy. Data were expressed as mean \pm SD. $* \mathrm{P}<0.05$ vs. Sham group; \#P $<0.05$ vs. SPRF group; \&P $<$ 0.05 vs. NFW group. One-way ANOVAs followed by the LSD test, $n=5$ per group.

\section{Supplementary Files}

This is a list of supplementary files associated with this preprint. Click to download.

- Additionalfile1.xls 